This item is the archived peer-reviewed author-version of:

\title{
Design, synthesis and biological evaluation of novel quinoline-based carboxylic hydrazides as anti-tubercular agents
}

\section{Reference:}

Chander Subhash, Ashok Penta, Cappoen Davie, Cos Paul, Murugesan Sankaranarayanan.- Design, synthesis and biological evaluation of novel quinoline-based carboxylic hydrazides as anti-tubercular agents

Chemical biology and drug design - ISSN 1747-0277 - 88:4(2016), p. 585-591

Full text (Publisher's DOI): http://dx.doi.org/doi:10.1111/CBDD.12788

To cite this reference: http://hdl.handle.net/10067/1351800151162165141 


\section{Design, synthesis and biological evaluation of novel quinoline based carboxylic hydrazides as anti-tubercular agents}

Subhash Chander ${ }^{1}$, Penta Ashok ${ }^{1}$, Cappoen Davie ${ }^{2}$, Paul Cos $^{2}$ and Sankaranarayanan Murugesan ${ }^{1, *}$

1,*Medicinal Chemistry Research Laboratory, Department of Pharmacy, Birla Institute of Technology \& Science, Pilani-333031. Rajasthan. India. E. mail: murugesan@pilani.bitspilani.ac.in

2, Laboratory of Microbiology, Parasitology and Hygiene (LMPH), S7, Faculty of Pharmaceutical, Biomedical and Veterinary Sciences, University of Antwerp, Universiteitsplein 1, B-2610 Wilrijk, Belgium.

Abstract: In the present study, seventeen novel quinoline based carboxylic hydrazides were designed as potential anti-tubercular agents using molecular hybridization approach and evaluated in-silico for drug likeness behaviour. The compounds were synthesized, purified and characterized using spectral techniques (like FTIR, ${ }^{1} \mathrm{H}$ NMR, and Mass). The in-vitro antitubercular activity (against $M$. tuberculosis $\mathrm{H} 37 \mathrm{Ra}$ ) and cytotoxicity against human lung fibroblast cells were studied. Among the tested hydrazides, four compounds $(\mathbf{6 h}, \mathbf{6 j}, \mathbf{6}$ and $\mathbf{6 m})$ exhibited significant anti-tubercular activity with MIC values below $20 \mu \mathrm{g} / \mathrm{mL}$. The two most potent compounds of the series, $6 \mathbf{j}$ and $\mathbf{6 m}$ exhibited MIC values 7.70 and $7.13 \mu \mathrm{g} / \mathrm{mL}$ respectively against $M$. tuberculosis with selectivity index greater than twenty six. Structure Activity Relationship (SAR) studies were performed for the tested compounds in order to explore the effect of substitution pattern on the anti-tubercular activity of the synthesized compounds.

Key words: Quinoline, Mycobacterium tuberculosis, drug likeness, cytotoxicity, fibroblast

\section{Introduction}

Tuberculosis (TB) is a pulmonary infectious disease with pandemic proportions. TB is caused by different members of Mycobacterium tuberculosis complex but, predominantly by Mycobacterium tuberculosis (Mtb). According to the WHO global tuberculosis report of 2015, over 9.6 million people fell ill and more than 1.5 million people died due to TB related complications in 2014 (1). 
Combination of multiple drugs (Drug Combo), is currently used for the treatment of TB. This drug combo speeds up the TB treatment as well as it reduces the chance of emerging drug resistance. In the treatment regimen, first line therapy includes oral combination of four key drugs (isoniazid, rifampicin, pyrazinamide and ethambutol) to which M. tuberculosis isolate is likely to be susceptible (2). Further, drugs like fluoroquinolones (levofloxacin generally preferred choice) and injectable drugs (generally used in the order: capreomycin, kanamycin, then amikacin) are preferentially given along with the first line drugs. Second-line drugs are used in the order: thioamides, cycloserine, then aminosalicylic acid. Furthermore, some reserve drugs like clofazimine, amoxicillin and clavulanate etc are less frequently used either due to their less effectiveness or lack of reliable clinical data. Overall, drug combination depends upon several factors like efficacy, safety, cost, patient's resistance profile, previous use of the drug etc (3). Although, drug combo is highly effective, but due to the lack of adherence to the therapy, increasing incidence of bacillary resistance including Multidrug-resistant TB (MDR-TB) and Extensively drug-resistant TB (XDR-TB) are frequently faced (4). In MDR-TB, bacteria becomes resistant to at least isoniazid and rifampicin, while in XDR TB in addition to rifampicin and isoniazid, bacteria gains resistant to one of the fluoroquinolones as well as one of the injectable drugs like kanamycin, amikacin or capreomycin. The treatment and current management of patients with MDR/XDR-TB is extremely complex for medical, social and public health systems $(5,6)$. Recently, emerging cases of totally drug-resistant (TDR) as well as co-infection of TB with HIV have lead to an increasing occurrence of treatment failure $(7,8)$. As a result, there is compiling need for the search of novel drugs active against both sensitive and drug resistant strains of Mtb, which can also act as a companion drug for existing treatment regimen of TB.

Quinoline based natural and synthetic compounds have privileged position in the medicinal chemistry due to their diverse biological activities and this moiety is frequently used in novel drug design (9-11). Among the diverse activities of quinoline, its anti-microbial activity is noteworthy. Quinoline acts as core pharmacophore in the several clinically approved drugs like mefloquine (12). It is interesting to note that, latest FDA fast track approved anti-TB drug bedaquiline (A, Figure 1) also known as TMC207 also possesses quinoline moiety (13). Bedaquiline possesses novel mechanism of action, it acts mainly by the inhibition of new target 
ATP synthase and exhibited potent activity against drug-sensitive as well as drug-resistant strains of M. tuberculosis with MIC 0.06 and $0.03 \mu \mathrm{g} / \mathrm{ml}$ respectively $(14,15)$.

Inspired by the discovery of Isoniazid (INH), a critical frontline drug in TB treatment, many isonicotinic hydrazones were investigated as potential antimycobacterial agents $(16,17)$. One of such hydrazones "verazide" (B, Figure 1) exhibited high anti-Mtb activity during the in-vitro studies (MIC $0.06 \mu \mathrm{g} / \mathrm{ml}$ ). Further, verazide exhibited high efficacy against TB induced lesions in the animal models (18).

By considering the above mentioned facts as well as our continuous interest to develop novel carboxylic hydrazide as anti-tubercular agents, in the present study, we used molecular hybridization approach to design novel pharmacophoric model $\mathbf{C}$ (Figure 1), consisting of carboxylic hydrazide subunit liked with quinoline moiety. Based upon the designed model $\mathbf{C}$, a series of compounds 6a-q (Table 2) were designed and in-silico evaluated for drug likeness properties (Table 1). Further, designed compounds were synthesized, characterized and evaluated for anti-Mtb activity as well as cytotoxicity. SAR of the reported novel compounds (6a-q) based upon the main scaffold $\mathbf{C}$ was investigated, in order to find new improved hits in terms of antitubercular potency and pharmacokinetic properties.

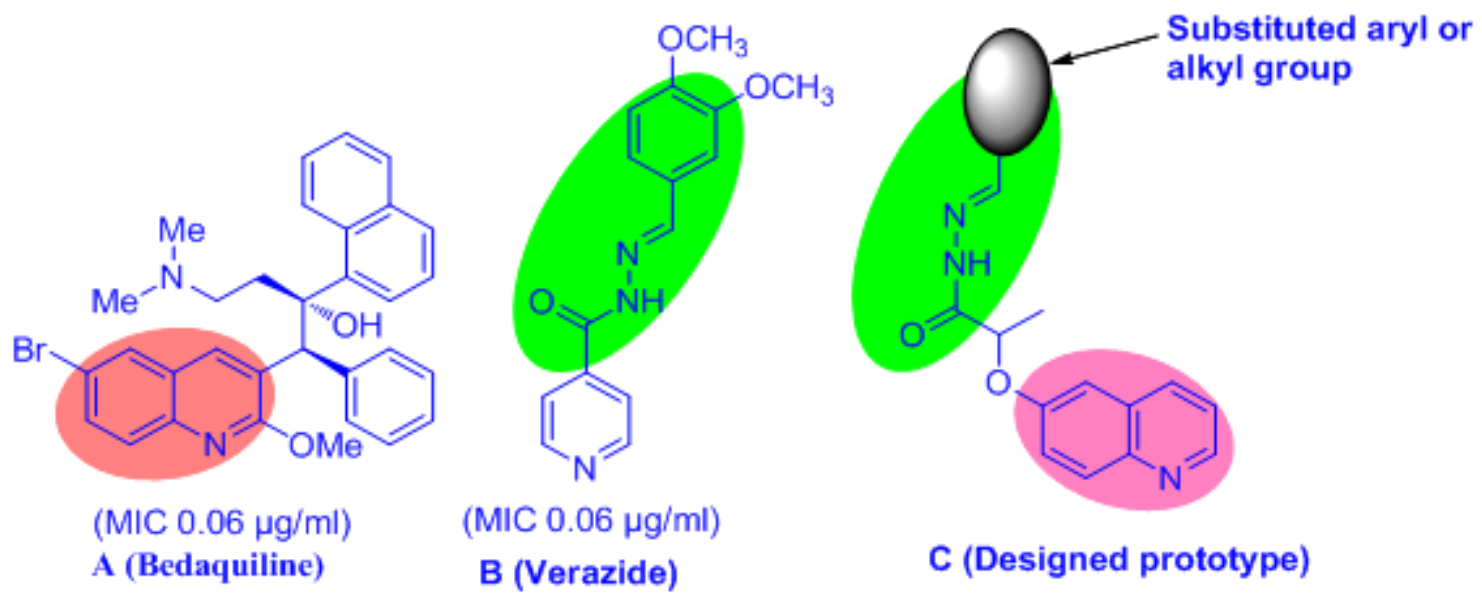

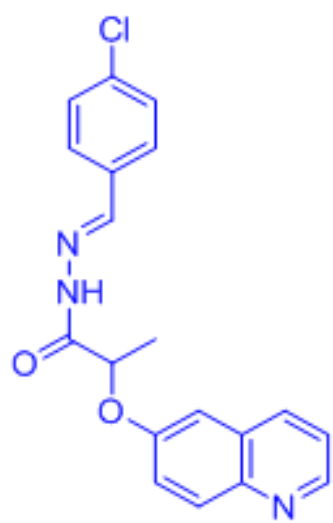

MIC $11.58 \mu \mathrm{g} / \mathrm{ml}$

$6 \mathrm{~h}$

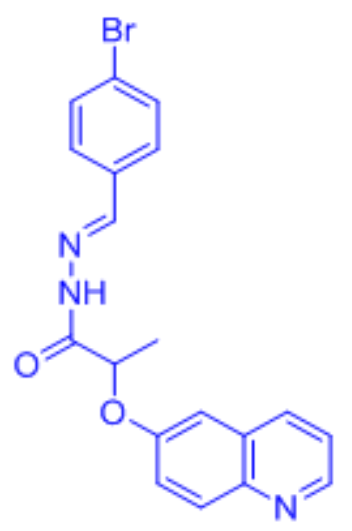

MIC $7.70 \mu \mathrm{g} / \mathrm{ml}$

$6 \mathrm{j}$

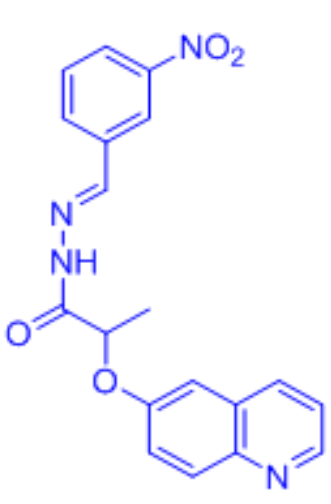

MIC $14.27 \mu \mathrm{g} / \mathrm{ml}$

61<smiles>CC(Oc1ccc2ncccc2c1)C(=O)N/N=C/c1ccc([N+](=O)[O-])cc1</smiles>

MIC $7.13 \mu \mathrm{g} / \mathrm{ml}$

$6 \mathrm{~m}$ 
Figure 1: Designing of novel anti-Mtb agents and structures of some significantly active compounds

\section{Experimental}

\section{In-silico prediction of drug likeness properties}

Physicochemical and pharmacokinetic parameters of the designed compounds were in-silico predicted using different tools like FAF-Drugs (19), Qikprop module of Schrödinger (20) and admetSAR (21). The different parameters predicted were; molecular weight, total solvent accessible surface area, octanol/water partition coefficient, aqueous solubility, number of rotatable bonds, number of hydrogen bond acceptor, number of hydrogen bond donor, acute oral toxicity, brain/blood partition coefficient, percentage human oral absorption and mutagenicity.

\section{Chemistry}

\section{Methods and Materials}

All reagents and solvents purchased from Sigma or SD fine companies were used as received without further purification. The progress of reaction was monitored by thin layer chromatography (TLC) using ethyl acetate and hexane (in suitable proportion) as mobile phase and silica as stationary phase. Melting points were uncorrected and determined in open capillary tubes on a Precision Buchi B530 (Flawil, Switzerland) melting point apparatus containing silicon oil. The IR spectra of the synthesized compounds were recorded using FTIR spectrophotometer (Shimadzu IR Prestige 21, Shimadzu, Mumbai, India). ${ }^{1} \mathrm{H}$ and ${ }^{13} \mathrm{C}$ NMR spectra were recorded on Bruker DPX-400 spectrometer (Bruker India Scientific Pvt. Ltd., Mumbai, India) using TMS as an internal standard (chemical shifts $\delta$ in ppm, $J$ values in $\mathrm{Hz}$ ). Elemental analysis was performed on Vario EL III M/s Elementar C, H, N, and S analyzer. ESI-MS were recorded on MICROMASS Quattro-II LCMS system (Waters Corporation, Milford, USA).

\section{General Chemistry}

All the synthesized compounds were primarily characterized by the non-spectral methods like TLC and melting points; further characterization was done by the spectral techniques like FTIR, ${ }^{1} \mathrm{H}$ NMR and Mass. Five representative compounds of the series (6c, $6 \mathbf{6 e}, \mathbf{6 i}, \mathbf{6 k}$ and $\left.\mathbf{6 q}\right)$ were also characterized by the ${ }^{13} \mathrm{C}$ NMR and elemental analysis. FTIR spectrum of the tested compounds exhibited the expected absorption bands, for example, all compounds possessed hydrogen at 
carboxylic hydrazide bond $(=\mathrm{N}-\mathrm{NH}-\mathrm{C}=\mathrm{O})$, a corresponding stretching peak (broad, moderate intensity) appeared in IR spectrum at $3175-3210 \mathrm{~cm}^{-1}$. Another discrete and characteristic peak (sharp, strong intensity), appeared at the region $1680-1703 \mathrm{~cm}^{-1}$ corresponding to the stretching of carbonyl group $(-\mathrm{NH}-\mathbf{C}=\mathbf{O})$. The ${ }^{1} \mathrm{H}$ NMR spectrum of the compounds showed, characteristic doublet around $\delta \sim 1.8$ corresponding to the methyl group (attached at the carbon connecting carbonyl and oxygen), while single proton at the adjacent carbon appeared as quartet $\delta \sim 5$. Further, proton at the $5^{\text {th }}$ position of quinoline showed characteristic peak $(\delta 7.15-7.20)$. Hydrazide proton shifted to most down field and appeared as singlet ( $\delta 9.30-10.04)$, further, single proton attached at the imine carbon also appeared at strong down field region $(\delta 8.80$ 8.90). The peak pattern and counting of NMR signals corresponding to the other protons of compounds were observed in compliance with the proposed structure. ${ }^{13} \mathrm{C}$ NMR data of the five representative compounds was also found in compliance with the proposed structure. The calculated and observed elemental values of $\mathrm{C} \mathrm{H} \mathrm{N}$ were found within the acceptable range. Mass spectrum (ESI-MS) of the synthesized compounds exhibited the corresponding M+1 peak.

\section{Synthesis of quinolin-6-ol (2)}

Commercially available compound, 6-methoxyquinoline 1 (7 g, $44 \mathrm{mmol}$ ) was added to stirred reaction mixture of aluminum trichloride $(17.6 \mathrm{~g}, 132 \mathrm{mmol})$ in $200 \mathrm{~mL}$ dry DCM. Further, reaction mass was allowed to stir at room temperature and progress of the reaction was monitored by TLC. After completion of reaction as per TLC (after twenty four hours), reaction mass was ice cooled and $50 \mathrm{~mL}$ cold water was added in order to quench the reaction and further stirred for twenty minutes. Then, reaction mass was transferred into separating funnel, $150 \mathrm{~mL}$ more distilled water was added, organic and aqueous layers were separated. Aqueous portion was further twice extracted with DCM $(2 \times 150 \mathrm{~mL})$, combined DCM portion was washed with brine $(400 \mathrm{~mL})$, dried over sodium sulphate and evaporated on rotary evaporator to afford the compound 2 (22).

Light brown solid; \%Yield 85; M.P. 146-148 ${ }^{\circ} \mathrm{C}$; IR (KBr, v, $\left.\mathrm{cm}^{-1}\right)$ : 3328, 3020, 2879, 1504, 1415, 1310, 1242, 1220, 921, 839; ${ }^{1} \mathrm{H}$ NMR (400 MHz, DMSO) $\delta 10.05$ (s, 1H), 8.66 (dd, $J=$ $4.1,1.6 \mathrm{~Hz}, 1 \mathrm{H}), 8.14(\mathrm{dd}, J=8.4,0.9 \mathrm{~Hz}, 1 \mathrm{H}), 7.87$ (d, $J=9.1 \mathrm{~Hz}, 1 \mathrm{H}), 7.40$ (dd, $J=8.3,4.2$ $\mathrm{Hz}, 1 \mathrm{H}), 7.32$ (dd, $J=9.1,2.7 \mathrm{~Hz}, 1 \mathrm{H}), 7.15$ (d, $J=2.7 \mathrm{~Hz}, 1 \mathrm{H}) ; \mathrm{MS}: \mathrm{m} / \mathrm{z} 146.1(\mathrm{M}+1)$.

\section{Synthesis of ethyl 2-(quinolin-6-yloxy)propanoate (3)}


To the stirred reaction mixture of intermediate $2(4.5 \mathrm{~g}, 31.02 \mathrm{mmol})$, potassium carbonate (10.7 $\mathrm{g}, 77.55 \mathrm{mmol})$ in $50 \mathrm{~mL} \mathrm{DMF}$, ethyl 2-bromopropanoate $(4.01 \mathrm{~mL}, 31.02 \mathrm{mmol})$ was added drop wise, and reaction was stirred at $100^{\circ} \mathrm{C}$, and progress was monitored by TLC. After completion of reaction as per TLC (after $10 \mathrm{~h}$ ), $150 \mathrm{~mL}$ ice-cold water was added to the reaction mass. Further, reaction mass was twice extracted with ethyl acetate $(2 \times 200 \mathrm{~mL})$ using separating funnel. Combined organic layer was washed with brine $(400 \mathrm{~mL})$, dried over anhydrous sodium sulphate and evaporated on rotary evaporator, which afforded titled compound $\mathbf{3}$ (23).

White solid; \%Yield 78; M.P. $128-130{ }^{\circ} \mathrm{C}$; IR $\left(\mathrm{KBr}, v, \mathrm{~cm}^{-1}\right)$ : 3116, 2991, 2756, 1747, 1504 , 1435, 1199, 1095, 1041, 968, 850; ${ }^{1} \mathrm{H}$ NMR $\left(400 \mathrm{MHz}, \mathrm{CDCl}_{3}\right) \delta 8.82(\mathrm{dd}, J=4.3,1.6 \mathrm{~Hz}, 1 \mathrm{H})$, $8.07(\mathrm{~d}, J=8.8 \mathrm{~Hz}, 2 \mathrm{H}), 7.48-7.38(\mathrm{~m}, 2 \mathrm{H}), 7.02(\mathrm{~d}, J=2.8 \mathrm{~Hz}, 1 \mathrm{H}), 4.91(\mathrm{q}, J=6.7 \mathrm{~Hz}, 1 \mathrm{H})$, $4.35-4.16(\mathrm{~m}, 2 \mathrm{H}), 1.72$ (d, $J=6.8 \mathrm{~Hz}, 3 \mathrm{H}), 1.26$ (t, $J=7.1 \mathrm{~Hz}, 3 \mathrm{H}) ; \mathrm{MS}: \mathrm{m} / \mathrm{z} 246.2(\mathrm{M}+1)$.

\section{Synthesis of 2-(quinolin-6-yloxy)propanehydrazide (4)}

To the stirred solution of intermediate $3(5.5 \mathrm{~g}, 22.44 \mathrm{mmol})$ in $80 \mathrm{~mL}$ ethanol, hydrazine hydrate (3.26 mL, $67.32 \mathrm{mmol}$ ) was added drop wise, followed by 1 drop of glacial acetic acid was added. Reaction mixture was refluxed and progress was monitored by TLC. After completion of reaction as per TLC (after $8 \mathrm{~h}$ refluxing), reaction mass was concentrated under reduced pressure, until it reduced to half of its original volume. Further, $100 \mathrm{~mL}$ of ice cold water was added, which resulted the precipitation of white solid. Finally, reaction mass was filtered using vacuum filtration, solid residues was thrice washed with distilled water $(3 \times 100 \mathrm{~mL})$ and dried to afford the titled compound 4 (24).

White solid; \%Yield 82; M.P. 118-120 ${ }^{\circ} \mathrm{C}$; IR $\left(\mathrm{KBr}, v, \mathrm{~cm}^{-1}\right): 3471,3055,2983,2904,2873$, 2785, 1681, 1514, 1505, 1237, 1334, 1178, 1097, 1041, 941; ${ }^{1} \mathrm{H}$ NMR (400 MHz, $\left.\mathrm{CDCl}_{3}\right) \delta 8.84$ (s, 1H), 8.07 (d, $J=8.4 \mathrm{~Hz}, 2 \mathrm{H}), 7.67(\mathrm{~s}, 1 \mathrm{H}), 7.46-7.36(\mathrm{~m}, 2 \mathrm{H}), 7.09$ (s, 1H), 4.96 (d, $J=6.6$ $\mathrm{Hz}, 1 \mathrm{H}), 3.90$ (s, 2H), 1.70 (d, $J=6.7 \mathrm{~Hz}, 3 \mathrm{H}) ; \mathrm{MS}: \mathrm{m} / \mathrm{z} 232.1(\mathrm{M}+1)$.

General procedure for the synthesis of quinolin-6-yloxy)propanehydrazide derivatives (6aq)

Intermediate hydrazide $4(0.231 \mathrm{~g}, 1 \mathrm{mmol})$ was added to the stirring solution of corresponding aldehydes $(1 \mathrm{mmol})$ in ethanol containing catalytic amount of glacial acetic acid. Reaction mixture was refluxed for 2 to $3 \mathrm{~h}$, progress of the reaction was monitored by TLC. After completion of reaction, ethanol was evaporated; ethyl acetate $(20 \mathrm{~mL})$ was added to the reaction 
mass. Ethyl acetate layer was twice washed with distilled water $(2 \times 20 \mathrm{~mL})$ and then with brine water $(20 \mathrm{~mL})$. Organic layer was dried over anhydrous sodium sulphate and finally evaporated on rotary evaporator to afford the final derivatives 6a-q (25). Synthesized compounds were purified by washing with hexane and cold diethyl ether.

\section{N'-benzylidene-2-(quinolin-6-yloxy)propanehydrazide (6a)}

White solid; \%Yield 82; M.P. 176-178 ${ }^{\circ} \mathrm{C}$; IR (KBr, v, $\left.\mathrm{cm}^{-1}\right)$ : 3194, 3076, 3051, 2978, 2904 , 1682, 1504, 1398, 1271, 1238, 1163, 1041; ${ }^{1} \mathrm{H}$ NMR (400 MHz, $\left.\mathrm{CDCl}_{3}\right) \delta 9.42(\mathrm{~s}, 1 \mathrm{H}), 8.84(\mathrm{~d}$, $J=2.7 \mathrm{~Hz}, 1 \mathrm{H}), 8.11-8.02(\mathrm{~m}, 2 \mathrm{H}), 7.54-7.46(\mathrm{~m}, 3 \mathrm{H}), 7.41(\mathrm{t}, J=7.5 \mathrm{~Hz}, 2 \mathrm{H}), 7.31(\mathrm{dd}, J=$ 7.5, $1.5 \mathrm{~Hz}, 2 \mathrm{H}) 7.14(\mathrm{~d}, J=2.8 \mathrm{~Hz}, 1 \mathrm{H}), 5.02(\mathrm{q}, J=6.6 \mathrm{~Hz}, 1 \mathrm{H}), 1.78(\mathrm{~d}, J=6.9 \mathrm{~Hz}, 3 \mathrm{H})$; MS: $\mathrm{m} / \mathrm{z} 320.1(\mathrm{M}+1)$.

\section{$N^{\prime}$-(2-methylbenzylidene)-2-(quinolin-6-yloxy)propanehydrazide (6b)}

White solid; \%Yield 86; M.P. 126-128 ${ }^{\circ} \mathrm{C}$; IR (KBr, v, $\left.\mathrm{cm}^{-1}\right): 3205,3055,2989,2908,1682$, 1556, 1504, 1293, 1222, 1078, 981, 848; ${ }^{1} \mathrm{H}$ NMR (400 MHz, $\left.\mathrm{CDCl}_{3}\right) \delta 9.65(\mathrm{~d}, J=5.7 \mathrm{~Hz}, 1 \mathrm{H})$, $8.83(\mathrm{~d}, J=2.9 \mathrm{~Hz}, 1 \mathrm{H}), 8.47(\mathrm{~s}, 1 \mathrm{H}), 8.09-8.06(\mathrm{~m}, 1 \mathrm{H}), 7.99(\mathrm{t}, J=8.8 \mathrm{~Hz}, 2 \mathrm{H}), 7.43-7.30$ $(\mathrm{m}, 3 \mathrm{H}), 7.22(\mathrm{~d}, J=7.3 \mathrm{~Hz}, 1 \mathrm{H}), 7.19(\mathrm{~d}, J=2.8 \mathrm{~Hz}, 1 \mathrm{H}), 7.15(\mathrm{~d}, J=7.5 \mathrm{~Hz}, 1 \mathrm{H}), 5.02(\mathrm{~d}, J=$ $6.8 \mathrm{~Hz}, 1 \mathrm{H}), 2.41(\mathrm{~s}, 3 \mathrm{H}), 1.81(\mathrm{~d}, J=5.4 \mathrm{~Hz}, 3 \mathrm{H}) ; \mathrm{MS}: \mathrm{m} / \mathrm{z} 334.2(\mathrm{M}+1)$.

\section{$N^{\prime}$-(3-methoxybenzylidene)-2-(quinolin-6-yloxy)propanehydrazide (6c)}

White solid; \%Yield 92; M.P. 142-144 ${ }^{\circ} \mathrm{C}$; IR $\left(\mathrm{KBr}, \mathrm{v}, \mathrm{cm}^{-1}\right): 3182,3084,3062,2983,2891$, $1681,1500,1452,1396,1273,1228,1136,1099,1039,933,829 ;{ }^{1} \mathrm{H} \mathrm{NMR}\left(400 \mathrm{MHz}, \mathrm{CDCl}_{3}\right) \delta$ $9.32(\mathrm{~s}, 1 \mathrm{H}), 8.84(\mathrm{~d}, J=2.7 \mathrm{~Hz}, 1 \mathrm{H}), 8.11-8.06(\mathrm{~m}, 3 \mathrm{H}), 7.63(\mathrm{~d}, J=8.0 \mathrm{~Hz}, 2 \mathrm{H}), 7.46(\mathrm{~d}, J=$ $9.4 \mathrm{~Hz}, 1 \mathrm{H}), 7.41(\mathrm{dd}, J=8.3,4.2 \mathrm{~Hz}, 1 \mathrm{H}), 7.21(\mathrm{~s}, 1 \mathrm{H}), 7.19(\mathrm{~d}, J=2.8 \mathrm{~Hz}, 2 \mathrm{H}), 5.02$ (q, $J=6.7$ $\mathrm{Hz}, 1 \mathrm{H}), 2.38(\mathrm{~s}, 3 \mathrm{H}), 1.79(\mathrm{~d}, J=6.8 \mathrm{~Hz}, 3 \mathrm{H}) ;{ }^{13} \mathrm{C} \mathrm{NMR}\left(100 \mathrm{MHz}, \mathrm{CDCl}_{3}\right) \delta 168.06,154.68$, $149.73,148.80,141.28,135.27,134.98,131.35,130.29,129.60,129.43,127.81,127.28,122.43$, 122.14, 121.76, 107.91, 75.39, 70.80, 21.57; Anal. calc. for $\mathrm{C}_{20} \mathrm{H}_{19} \mathrm{~N}_{3} \mathrm{O}_{3}: \mathrm{C}, 68.75 ; \mathrm{H}, 5.48 ; \mathrm{N}$, 12.03. Found: C, 68.93; H, 5.41; N, 12.11; MS: m/z $350.1(\mathrm{M}+1)$.

\section{$N^{\prime}$-(4-methoxybenzylidene)-2-(quinolin-6-yloxy)propanehydrazide (6d)}


White solid; \%Yield 87; M.P. 144-146 ${ }^{\circ} \mathrm{C}$; IR (KBr, v, $\left.\mathrm{cm}^{-1}\right)$ : 3178, 3057, 2900, 2872, 2559, 1681, 1504, 1311, 1274, 1226, 1180, 798; ${ }^{1} \mathrm{H}$ NMR (400 MHz, $\left.\mathrm{CDCl}_{3}\right) \delta 9.30$ (s, 1H), 8.84 (dd, $J=4.2,1.6 \mathrm{~Hz}, 1 \mathrm{H}), 8.11-8.01(\mathrm{~m}, 3 \mathrm{H}), 7.72-7.65(\mathrm{~m}, 2 \mathrm{H}), 7.43(\mathrm{ddd}, J=14.8,8.7,3.5 \mathrm{~Hz}$, 2H), $7.18(\mathrm{~d}, J=2.8 \mathrm{~Hz}, 1 \mathrm{H}), 6.91(\mathrm{~d}, J=8.9 \mathrm{~Hz}, 2 \mathrm{H}), 5.01(\mathrm{q}, J=6.7 \mathrm{~Hz}, 1 \mathrm{H}), 3.85(\mathrm{~s}, 3 \mathrm{H})$, $1.79(\mathrm{~d}, J=6.8 \mathrm{~Hz}, 3 \mathrm{H}) ; \mathrm{MS}: \mathrm{m} / \mathrm{z} 350.1(\mathrm{M}+1)$.

\section{$N^{\prime}$-(2-fluorobenzylidene)-2-(quinolin-6-yloxy)propanehydrazide (6e)}

White solid; \%Yield 87; M.P. 148-150 ${ }^{\circ} \mathrm{C}$; IR (KBr, v, $\left.\mathrm{cm}^{-1}\right)$ : 3198, 3068, 2983, 2970, 2864, 1691, 1500, 1452, 1381, 1269, 1222, 1093, 1039, 972; ${ }^{1} \mathrm{H}$ NMR (400 MHz, $\left.\mathrm{CDCl}_{3}\right) \delta 9.57(\mathrm{~s}$, $1 \mathrm{H}), 8.84(\mathrm{dd}, J=4.2,1.5 \mathrm{~Hz}, 1 \mathrm{H}), 8.39$ (s, 1H), 8.09 (ddd, $J=9.4,5.6,4.0 \mathrm{~Hz}, 3 \mathrm{H}), 7.47-7.36$ (m, 3H), $7.21-7.15(\mathrm{~m}, 2 \mathrm{H}), 7.09-7.04(\mathrm{~m}, 1 \mathrm{H}), 5.03$ (q, $J=6.8 \mathrm{~Hz}, 1 \mathrm{H}), 1.79$ (d, $J=6.8 \mathrm{~Hz}$, $3 \mathrm{H}) ;{ }^{13} \mathrm{C}$ NMR $\left(100 \mathrm{MHz}, \mathrm{CDCl}_{3}\right) \delta 168.30,161.49$ (d, $\left.J=252.5 \mathrm{~Hz}\right), 154.62,148.83,144.77$, 142.61, 135.23, 132.36 (d, $J=8.5 \mathrm{~Hz}), 131.39,129.09,127.30,124.47$ (d, $J=3.5 \mathrm{~Hz}), 122.39$,

122.11, 121.77, $115.64(\mathrm{~d}, J=20.9 \mathrm{~Hz}), 107.92,75.32,19.04$; Anal. calc. for $\mathrm{C}_{19} \mathrm{H}_{16} \mathrm{FN}_{3} \mathrm{O}_{2}$ : C, 67.65; H, 4.78; N, 12.46. Found: C, 67.47; H, 4.83; N, 12.57; MS: m/z 338.7 (M+1).

\section{$N^{\prime}$-(4-fluorobenzylidene)-2-(quinolin-6-yloxy)propanehydrazide (6f)}

White solid; \%Yield 91; M.P. 162-164 ${ }^{\circ} \mathrm{C}$; IR (KBr, v, $\left.\mathrm{cm}^{-1}\right)$ : 3188, 3076, 3057, 2987, 2962, 2781, 1681, 1504, 1454, 1404, 1273, 1232, 1159, 1096, 1039; ${ }^{1} \mathrm{H}$ NMR (400 MHz, $\left.\mathrm{CDCl}_{3}\right) \delta$ $9.49(\mathrm{~s}, 1 \mathrm{H}), 8.89-8.80(\mathrm{~m}, 1 \mathrm{H}), 8.15(\mathrm{~s}, 1 \mathrm{H}), 8.09-8.03(\mathrm{~m}, 2 \mathrm{H}), 7.72(\mathrm{dd}, J=8.7,5.4 \mathrm{~Hz}$, 2H), $7.46-7.38(\mathrm{~m}, 2 \mathrm{H}), 7.18(\mathrm{~d}, J=2.7 \mathrm{~Hz}, 1 \mathrm{H}), 7.08(\mathrm{t}, J=8.7 \mathrm{~Hz}, 2 \mathrm{H}), 5.02(\mathrm{q}, J=6.8 \mathrm{~Hz}$, 1H), 1.79 (d, $J=6.8 \mathrm{~Hz}, 3 \mathrm{H}) ; \mathrm{MS}: \mathrm{m} / \mathrm{z} 338.7(\mathrm{M}+1)$.

\section{N'-(2-chlorobenzylidene)-2-(quinolin-6-yloxy)propanehydrazide (6g)}

White solid; \%Yield 86; M.P. 166-168 ${ }^{\circ} \mathrm{C}$; IR (KBr, v, $\left.\mathrm{cm}^{-1}\right)$ : 3196, 3055, 2989, 2937, 1686, 1552, 1504, 1469, 1284, 1234, 1130, 1049, 981, 927; ${ }^{1} \mathrm{H}$ NMR (400 MHz, $\left.\mathrm{CDCl}_{3}\right) \delta 9.47$ (s, 1H), 8.80 (dd, $J=4.2,1.5 \mathrm{~Hz}, 1 \mathrm{H}), 8.37$ (s, 1H), 8.07 (ddd, $J=9.2,5.4,4.0 \mathrm{~Hz}, 3 \mathrm{H}), 7.43-7.31$ (m, 3H), $7.19-7.13(\mathrm{~m}, 2 \mathrm{H}), 7.07-7.01(\mathrm{~m}, 1 \mathrm{H}), 5.02(\mathrm{q}, J=6.8 \mathrm{~Hz}, 1 \mathrm{H}), 1.77(\mathrm{~d}, J=6.8 \mathrm{~Hz}, 3 \mathrm{H})$; MS: m/z $354.2(\mathrm{M}+1)$. 
White solid; \%Yield 89; M.P. $178-180{ }^{\circ} \mathrm{C}$; IR $\left(\mathrm{KBr}, v, \mathrm{~cm}^{-1}\right)$ : 3186, 3067, 3062, 2987, 2872, 2831, 2603, 1681, 1498, 1454, 1409, 1261, 1230, 1136, 1097, 1041; ${ }^{1} \mathrm{H}$ NMR (400 MHz, $\left.\mathrm{CDCl}_{3}\right) \delta 9.53(\mathrm{~s}, 1 \mathrm{H}), 8.84(\mathrm{~d}, J=3.0 \mathrm{~Hz}, 1 \mathrm{H}), 8.15(\mathrm{~s}, 1 \mathrm{H}), 8.05(\mathrm{dd}, J=16.3,6.8 \mathrm{~Hz}, 2 \mathrm{H})$, $7.66(\mathrm{~d}, J=8.5 \mathrm{~Hz}, 2 \mathrm{H}), 7.43-7.34(\mathrm{~m}, 4 \mathrm{H}), 7.17(\mathrm{~d}, J=2.7 \mathrm{~Hz}, 1 \mathrm{H}), 5.02(\mathrm{q}, J=6.8 \mathrm{~Hz}, 1 \mathrm{H})$, $1.79(\mathrm{~d}, J=6.8 \mathrm{~Hz}, 3 \mathrm{H}) ; \mathrm{MS}: \mathrm{m} / \mathrm{z} 354.2(\mathrm{M}+1)$.

\section{$N^{\prime}$-(3-bromobenzylidene)-2-(quinolin-6-yloxy)propanehydrazide (6i)}

White solid; \%Yield 94; M.P. 108-110 ${ }^{\circ} \mathrm{C}$; IR $\left(\mathrm{KBr}, v, \mathrm{~cm}^{-1}\right)$ : 3195, 3049, 2831, 2357, 1682 , 1500, 1498, 1288, 1172, 1068, 784; ${ }^{1} \mathrm{H}$ NMR $\left(400 \mathrm{MHz}, \mathrm{CDCl}_{3}\right) \delta 9.56(\mathrm{~s}, 1 \mathrm{H}), 8.85(\mathrm{~s}, 1 \mathrm{H})$, $8.13(\mathrm{~s}, 1 \mathrm{H}), 8.07$ (t, $J=9.9 \mathrm{~Hz}, 2 \mathrm{H}), 7.93(\mathrm{~s}, 1 \mathrm{H}), 7.55(\mathrm{dt}, J=17.1,8.6 \mathrm{~Hz}, 3 \mathrm{H}), 7.46-7.35$ $(\mathrm{m}, 2 \mathrm{H}), 7.18(\mathrm{~s}, 1 \mathrm{H}), 5.02(\mathrm{q}, J=6.6 \mathrm{~Hz}, 1 \mathrm{H}), 1.79(\mathrm{~d}, J=6.9 \mathrm{~Hz}, 3 \mathrm{H}) ;{ }^{13} \mathrm{C} \mathrm{NMR}(100 \mathrm{MHz}$, $\left.\mathrm{CDCl}_{3}\right) \delta 168.19,154.56,147.84,135.22,133.67,132.45,131.54,130.19,130.25,128.16$, $126.50,126.14,122.97,122.07,119.71,108.04,107.30,75.37,18.99$; Anal. calc. for $\mathrm{C}_{19} \mathrm{H}_{16} \mathrm{BrN}_{3} \mathrm{O}_{2}$ : C, 57.30; H, 4.05; N, 10.55. Found: C, 57.72; H, 4.09; N, 10.37; MS: m/z 398.1 $(\mathrm{M}+1)$.

\section{$N^{\prime}$-(4-bromobenzylidene)-2-(quinolin-6-yloxy)propanehydrazide (6j)}

White solid; \%Yield 88; M.P. 162-164 ${ }^{\circ} \mathrm{C}$; IR (KBr, v, $\left.\mathrm{cm}^{-1}\right)$ : 3197, 3086, 2987, 2908, 2873 , 2829, 2785, 2603, 1681, 1622, 1498, 1450, 1398, 1230, 1095, 754; ${ }^{1} \mathrm{H}$ NMR (400 MHz, $\left.\mathrm{CDCl}_{3}\right)$ $\delta 9.55(\mathrm{~s}, 1 \mathrm{H}), 8.84(\mathrm{~d}, J=3.0 \mathrm{~Hz}, 1 \mathrm{H}), 8.15(\mathrm{~s}, 1 \mathrm{H}), 8.09-8.03(\mathrm{~m}, 2 \mathrm{H}), 7.58(\mathrm{~d}, J=8.6 \mathrm{~Hz}$, 2H), $7.53-7.50(\mathrm{~m}, 2 \mathrm{H}), 7.43-7.37(\mathrm{~m}, 2 \mathrm{H}), 7.17(\mathrm{~d}, J=2.8 \mathrm{~Hz}, 1 \mathrm{H}), 5.01(\mathrm{q}, J=6.8 \mathrm{~Hz}, 1 \mathrm{H})$, $1.78(\mathrm{~d}, J=6.7 \mathrm{~Hz}, 3 \mathrm{H}) ; \mathrm{MS}: \mathrm{m} / \mathrm{z} 398.1(\mathrm{M}+1)$.

\section{$N^{\prime}$-(2-nitrobenzylidene)-2-(quinolin-6-yloxy)propanehydrazide (6k)}

Light yellow solid; \%Yield 91; M.P. 158-160 ${ }^{\circ} \mathrm{C}$; IR (KBr, v, $\left.\mathrm{cm}^{-1}\right)$ : 3197, 3159, 3053, 2868, 1703, 1519, 1504, 1440, 1338, 1274, 1222, 1172, 1080, 979, 840, 754; ${ }^{1} \mathrm{H}$ NMR (400 MHz, $\left.\mathrm{CDCl}_{3}\right) \delta 10.04(\mathrm{~s}, 1 \mathrm{H}), 8.83(\mathrm{~d}, J=4.0 \mathrm{~Hz}, 1 \mathrm{H}), 8.77(\mathrm{~s}, 1 \mathrm{H}), 8.27(\mathrm{~d}, J=7.9 \mathrm{~Hz}, 1 \mathrm{H}), 8.08(\mathrm{~d}, J$ $=8.3 \mathrm{~Hz}, 1 \mathrm{H}), 8.02(\mathrm{dd}, J=13.9,8.7 \mathrm{~Hz}, 2 \mathrm{H}), 7.66(\mathrm{t}, J=7.8 \mathrm{~Hz}, 1 \mathrm{H}), 7.55(\mathrm{t}, J=7.7 \mathrm{~Hz}, 1 \mathrm{H})$, $7.41(\mathrm{dd}, J=8.3,4.2 \mathrm{~Hz}, 1 \mathrm{H}), 7.36(\mathrm{dd}, J=9.2,2.7 \mathrm{~Hz}, 1 \mathrm{H}), 7.19(\mathrm{~d}, J=2.6 \mathrm{~Hz}, 1 \mathrm{H}), 5.05(\mathrm{q}, J$ $=6.8 \mathrm{~Hz}, 1 \mathrm{H}), 1.80(\mathrm{~d}, J=6.7 \mathrm{~Hz}, 3 \mathrm{H}) ;{ }^{13} \mathrm{C} \mathrm{NMR}\left(100 \mathrm{MHz}, \mathrm{CDCl}_{3}\right) \delta 168.54,154.52,148.89$, 148.07, 144.82, 144.61, 135.23, 133.67, 131.51, 130.86, 129.28, 129.08, 128.30, 124.80, 122.09, 
121.79, 108.07, 75.28, 18.92; Anal. calc. for $\mathrm{C}_{19} \mathrm{H}_{16} \mathrm{~N}_{4} \mathrm{O}_{4}$ : C, 62.63; H, 4.43; N, 15.38. Found: C, 62.91; H, 4.47; N, 15.29; MS: m/z 365.1 (M+1).

\section{$N^{\prime}$-(3-nitrobenzylidene)-2-(quinolin-6-yloxy)propanehydrazide (6l)}

Light yellow solid; \%Yield 88; M.P. $168-170{ }^{\circ} \mathrm{C}$; IR (KBr, v, $\left.\mathrm{cm}^{-1}\right)$ : 3196, 3161, 3041, 3028, 1666, 1529, 1504, 1288, 1224, 1078, 981, 850; ${ }^{1} \mathrm{H}$ NMR (400 MHz, $\left.\mathrm{CDCl}_{3}\right) \delta 9.67$ (s, 1H), 8.85 $(\mathrm{dd}, J=4.2,1.5 \mathrm{~Hz}, 1 \mathrm{H}), 8.50(\mathrm{~s}, 1 \mathrm{H}), 8.38(\mathrm{~s}, 1 \mathrm{H}), 8.26(\mathrm{dd}, J=8.2,1.3 \mathrm{~Hz}, 1 \mathrm{H}), 8.15-8.03$ (m, 3H), $7.60(\mathrm{t}, J=8.0 \mathrm{~Hz}, 1 \mathrm{H}), 7.42(\mathrm{dt}, J=9.2,3.4 \mathrm{~Hz}, 2 \mathrm{H}), 7.19$ (d, $J=2.7 \mathrm{~Hz}, 1 \mathrm{H}), 5.05(\mathrm{q}$, $J=6.8 \mathrm{~Hz}, 1 \mathrm{H}), 1.81(\mathrm{~d}, J=6.8 \mathrm{~Hz}, 3 \mathrm{H}) ; \mathrm{MS}: \mathrm{m} / \mathrm{z} 365.1(\mathrm{M}+1)$.

\section{$N^{\prime}$-(4-nitrobenzylidene)-2-(quinolin-6-yloxy)propanehydrazide (6m)}

Light yellow solid; \%Yield 92; M.P. 134-136 ${ }^{\circ} \mathrm{C}$; IR (KBr, v, $\left.\mathrm{cm}^{-1}\right)$ : 3213, 3032, 2983, 1683, 1681, 1517, 1504, 1338, 1224, 1089, 968; ${ }^{1} \mathrm{H}$ NMR (400 MHz, $\left.\mathrm{CDCl}_{3}\right) \delta 9.91(\mathrm{~s}, 1 \mathrm{H}), 8.84(\mathrm{~d}, J$ $=3.7 \mathrm{~Hz}, 1 \mathrm{H}), 8.36(\mathrm{~s}, 1 \mathrm{H}), 8.23(\mathrm{~d}, J=8.2 \mathrm{~Hz}, 2 \mathrm{H}), 8.08(\mathrm{~d}, J=8.4 \mathrm{~Hz}, 1 \mathrm{H}), 7.99(\mathrm{~d}, J=9.2$ $\mathrm{Hz}, 1 \mathrm{H}), 7.89$ (t, $J=10.9 \mathrm{~Hz}, 2 \mathrm{H}), 7.42(\mathrm{dd}, J=8.1,4.0 \mathrm{~Hz}, 1 \mathrm{H}), 7.33(\mathrm{~d}, J=7.1 \mathrm{~Hz}, 1 \mathrm{H}), 7.17$ (s, 1H), $5.04(\mathrm{q}, J=6.6 \mathrm{~Hz}, 1 \mathrm{H}), 1.80(\mathrm{~d}, J=6.7 \mathrm{~Hz}, 3 \mathrm{H}) ; \mathrm{MS}: \mathrm{m} / \mathrm{z} 365.1(\mathrm{M}+1)$.

\section{$N^{\prime}$-(4-cyanobenzylidene)-2-(quinolin-6-yloxy)propanehydrazide (6n)}

White solid; \%Yield 83; M.P. 174-176 ${ }^{\circ} \mathrm{C}$; IR $\left(\mathrm{KBr}, \mathrm{v}, \mathrm{cm}^{-1}\right)$ : 3250, 3051, 3010, 2225, 1697 , 1500, 1319, 1253, 1228, 1136, 936; ${ }^{1} \mathrm{H}$ NMR $\left(400 \mathrm{MHz}, \mathrm{CDCl}_{3}\right) \delta 9.81(\mathrm{~s}, 1 \mathrm{H}), 8.84(\mathrm{~d}, J=2.9$ $\mathrm{Hz}, 1 \mathrm{H}), 8.29$ (s, 1H), 8.08 (d, $J=7.8 \mathrm{~Hz}, 1 \mathrm{H}), 7.99$ (d, $J=9.2 \mathrm{~Hz}, 1 \mathrm{H}), 7.82(\mathrm{~d}, J=8.3 \mathrm{~Hz}, 2 \mathrm{H})$, $7.67(\mathrm{~d}, J=8.4 \mathrm{~Hz}, 2 \mathrm{H}), 7.42(\mathrm{dd}, J=8.3,4.2 \mathrm{~Hz}, 1 \mathrm{H}), 7.34(\mathrm{dd}, J=9.2,2.8 \mathrm{~Hz}, 1 \mathrm{H}), 7.16(\mathrm{~d}, J$ $=2.7 \mathrm{~Hz}, 1 \mathrm{H}), 5.03(\mathrm{q}, J=6.8 \mathrm{~Hz}, 1 \mathrm{H}), 1.79(\mathrm{~d}, J=6.8 \mathrm{~Hz}, 3 \mathrm{H}) ; \mathrm{MS}: \mathrm{m} / \mathrm{z} 345.3(\mathrm{M}+1)$.

\section{$N^{\prime}$-(3,4-dichlorobenzylidene)-2-(quinolin-6-yloxy)propanehydrazide (6o)}

White solid; \%Yield 78; M.P. 170-172 ${ }^{\circ} \mathrm{C}$; IR (KBr, v, $\left.\mathrm{cm}^{-1}\right)$ : 3176, 3024, 2993, 2902, 2829 , 2600, 1693, 1554, 1498, 1471, 1377, 1325, 1222, 1128; ${ }^{1} \mathrm{H}$ NMR $\left(400 \mathrm{MHz}, \mathrm{CDCl}_{3}\right) \delta 9.67(\mathrm{~s}$, $1 \mathrm{H}), 8.84(\mathrm{~s}, 1 \mathrm{H}), 8.16(\mathrm{~s}, 1 \mathrm{H}), 8.07(\mathrm{~d}, J=8.3 \mathrm{~Hz}, 1 \mathrm{H}), 8.01(\mathrm{~d}, J=9.4 \mathrm{~Hz}, 1 \mathrm{H}), 7.83(\mathrm{~s}, 1 \mathrm{H})$, $7.56-7.43(\mathrm{~m}, 3 \mathrm{H}), 7.39-7.33(\mathrm{~m}, 1 \mathrm{H}), 7.17(\mathrm{~s}, 1 \mathrm{H}), 5.02(\mathrm{q}, J=6.4 \mathrm{~Hz}, 1 \mathrm{H}), 1.79(\mathrm{~d}, J=6.6$ $\mathrm{Hz}, 3 \mathrm{H}) ; \mathrm{MS}: \mathrm{m} / \mathrm{z} 388.2(\mathrm{M}+1)$. 


\section{2-(quinolin-6-yloxy)-N'-(3,4,5-trimethoxybenzylidene)propanehydrazide (6p)}

White solid; \%Yield 73; M.P. 180-182 ${ }^{\circ} \mathrm{C}$; IR (KBr, v, $\left.\mathrm{cm}^{-1}\right)$ : 3201, 3051, 2935, 2902, 2883 , 2642, 1681, 1504, 1415, 1330, 1232, 1126, 1008, 943; ${ }^{1} \mathrm{H}$ NMR $\left(400 \mathrm{MHz}, \mathrm{CDCl}_{3}\right) \delta 9.49(\mathrm{~s}$, 1H), $8.90-8.80(\mathrm{~m}, 1 \mathrm{H}), 8.11-8.02(\mathrm{~m}, 3 \mathrm{H}), 7.45-7.37$ (m, 2H), 7.17 (s, 1H), $6.92(\mathrm{dd}, J=$ 16.0, $1.7 \mathrm{~Hz}, 2 \mathrm{H}), 5.07-4.96(\mathrm{~m}, 1 \mathrm{H}), 3.88(\mathrm{~d}, J=1.6 \mathrm{~Hz}, 9 \mathrm{H}), 1.79$ (d, $J=6.8 \mathrm{~Hz}, 3 \mathrm{H})$.

; MS: $\mathrm{m} / \mathrm{z} 410.3(\mathrm{M}+1)$.

\section{$N^{\prime}$-(2-methylpropylidene)-2-(quinolin-6-yloxy)propanehydrazide (6q)}

White solid; \%Yield 78; M.P. 132-134 ${ }^{\circ} \mathrm{C}$; IR (KBr, v, $\left.\mathrm{cm}^{-1}\right)$ : 3188, 3061, 2962, 2924, 2870 , 2796, 1680, 1502, 1381, 1321, 1242, 1226, 1134, 1055, 968; ${ }^{1} \mathrm{H}$ NMR (400 MHz, $\left.\mathrm{CDCl}_{3}\right) \delta 9.12$ (s, 1H), 8.83 (dd, $J=4.2,1.6 \mathrm{~Hz}, 1 \mathrm{H}), 8.11-8.02(\mathrm{~m}, 2 \mathrm{H}), 7.44-7.36(\mathrm{~m}, 2 \mathrm{H}), 7.32$ (d, $J=6.1$ $\mathrm{Hz}, 1 \mathrm{H}), 7.14(\mathrm{~d}, J=2.8 \mathrm{~Hz}, 1 \mathrm{H}), 4.95(\mathrm{q}, J=6.8 \mathrm{~Hz}, 1 \mathrm{H}), 2.71-2.63(\mathrm{~m}, 1 \mathrm{H}), 1.76-1.73(\mathrm{~m}$, $3 \mathrm{H}), 1.11(\mathrm{dd}, J=11.8,6.9 \mathrm{~Hz}, 6 \mathrm{H}) .{ }^{13} \mathrm{C} \mathrm{NMR}\left(100 \mathrm{MHz}, \mathrm{CDCl}_{3}\right) \delta 167.91,158.52,154.65$, 148.80, 135.25, 131.31, 129.10, 122.10, 121.77, 107.85, 75.24, 31.65, 19.74, 19.02. Anal. calc. for $\mathrm{C}_{16} \mathrm{H}_{19} \mathrm{~N}_{3} \mathrm{O}_{2}: \mathrm{C}, 67.35 ; \mathrm{H}, 6.71 ; \mathrm{N}, 14.73$. Found: $\mathrm{C}, 67.56 ; \mathrm{H}, 6.67 ; \mathrm{N}, 14.61 ; \mathrm{MS}: \mathrm{m} / \mathrm{z} 286.2$ $(\mathrm{M}+1)$.

\section{Biological evaluation}

\section{Evaluation of anti-tubercular activity}

The Minimal Inhibitory Concentration (MIC) against mycobacteria of all the synthesized compounds was evaluated by serial dilution method. The in vitro assay was based on a method in which a luminescent Mycobacterium tuberculosis H37Ra strain Lehmann \&Neumann (ATCC ${ }^{\circ}$ $25177^{\mathrm{TM}}$ ) transformed with pSMT1 luciferase reporter plasmid is used. The tested compounds were solubilized in DMSO (Sigma-Aldrich) at stock concentration of $10 \mathrm{mM}$. Serial dilutions of each compound were made in liquid 7H9 medium [Middlebrook 7H9 broth based (Difco)] with $10 \%$ oleic acid, albumin, dextrose, catalase (OADC) enrichment. Volumes of $20 \mu \mathrm{L}$ of the serial dilutions were added in triplicate to 96 well, flat-bottomed micro well plates. The bacterial suspension was made by thawing and dissolving a frozen Mycobacteria pellet in 7H9-10\% OADC. The dissolved pellet was passed through a $5.0 \mu \mathrm{M}$ filter (Millipore) to eliminate clumps and left for 1 hour to recover at $37{ }^{\circ} \mathrm{C}, 5 \% \mathrm{CO}_{2}$. Next, the bacterial suspension was diluted in 7H9-10\% OADC to obtain 50,000 Relative Light Units (RLU)/mL and a volume of $180 \mu \mathrm{L}$ of 
bacteria was added to each well. A bacterial replication was analyzed by luminometry after 6 days of incubation. The bacterial suspension from each well was collected and transferred to a black 96-well plate to evade cross luminescence between wells. The luminescent signal was evoked by addition of the substrate for the bacterial luciferase, $1 \%$ n-decanal in ethanol to each well by the Discover multi-plate reader from Promega and the light emission in each well was measured (26).

\section{Cytotoxicity evaluation}

Cytotoxic effects on the MRC-5, human lung fibroblast cell (ATCC@ CCL-171 ${ }^{\mathrm{TM}}$ ) was determined for the derivatives by a neutral red uptake assay. The neutral red uptake assay relies on the ability of viable cells to bind and incorporate the neutral red dye (toluylene red). The acute toxic concentration $\left(\mathrm{IC}_{50}\right)$ of a compound is defined as the concentration at which the uptake of the neutral red dye by the cells is reduced by $50 \%$. The MRC- 5 cells were grown in Dulbecco's modified Eagle's medium (DMEM) with 10\% Foetal calf serum (FCS) until a semi-confluent layer of cells was obtained. The cells were trypsinized, washed and 40000 cells were seeded per well of a 96-well plate and left for recovery at $37^{\circ} \mathrm{C}, 5 \% \mathrm{CO}_{2}$. The following days, the compounds were solublized in DMSO to stock concentrations of $10 \mu \mathrm{M}$. A serial dilution of each compound was made in DMEM with 10\% FCS. The MRC-5 cells were washed and exposed to the derivatives by adding the serial dilutions of the compounds to the wells. The plates were left for incubation at $37^{\circ} \mathrm{C}, 5 \% \mathrm{CO}_{2}$ for $24 \mathrm{~h}$. After exposure, the cells were washed with $200 \mu \mathrm{L}$ of PBS, and $200 \mu \mathrm{L}$ of neutral red working solution (Sigma) was added to each well. Subsequently, the plates were incubated for $3 \mathrm{~h}$ at $37^{\circ} \mathrm{C}, 5 \% \mathrm{CO}_{2}$. The wells were washed with $200 \mu \mathrm{L}$ of PBS and $200 \mu \mathrm{L}$ of ethanol/acetic acid (50\%) mixture. The plates were left on the shaker until the color became homogeneous purple and the optical density was measured at $530 \mathrm{~nm}$ (NR max) and $620 \mathrm{nM}$ (reference wavelength) with the Paradigm detection platform (27).

\section{Results and Discussion}

\section{In-silico prediction of drug likeness properties}

The values of in-silico predicted physicochemical and pharmacokinetic parameters of the designed compounds are shown in Table 1, while their range such parameters followed by $95 \%$ of known drugs are also shown on the footnotes of Table $\mathbf{1}(28,29)$. The results of the predicted 
drug-likeness parameters revealed that, value of descriptors like mol. wt, HBA, HBD and $\log \mathrm{P}$ followed Lipinski rule of five and their values lied within the prescribed range. Compound 60 may have less aqueous solubility, due to its low value of predicted $\log \mathrm{S}$, but still it lied within the range (-6.5 to 0.5). Furthermore, descriptors like SASA, Rot, $\log B \mathrm{~B}$ were also found within the range. Predicted acute oral toxicity values of all the compounds lie in "class III" which revealed that, compounds possessed fairly high lethal dose and can be considered suitable for the druggable point of view. All designed compounds exhibited good predicted values of percentage human oral absorption (except compounds $6 \mathbf{l}$ and $\mathbf{6 m}$ ) and none of the compound possessed mutagenicity (predicted in qualitative terms). So overall, based upon the values of predicted drug likeness parameters, all compounds possessed the drug-likeness behavior.

Table 1: In-silico predicted physicochemical and pharmacokinetic parameters of the designed compounds

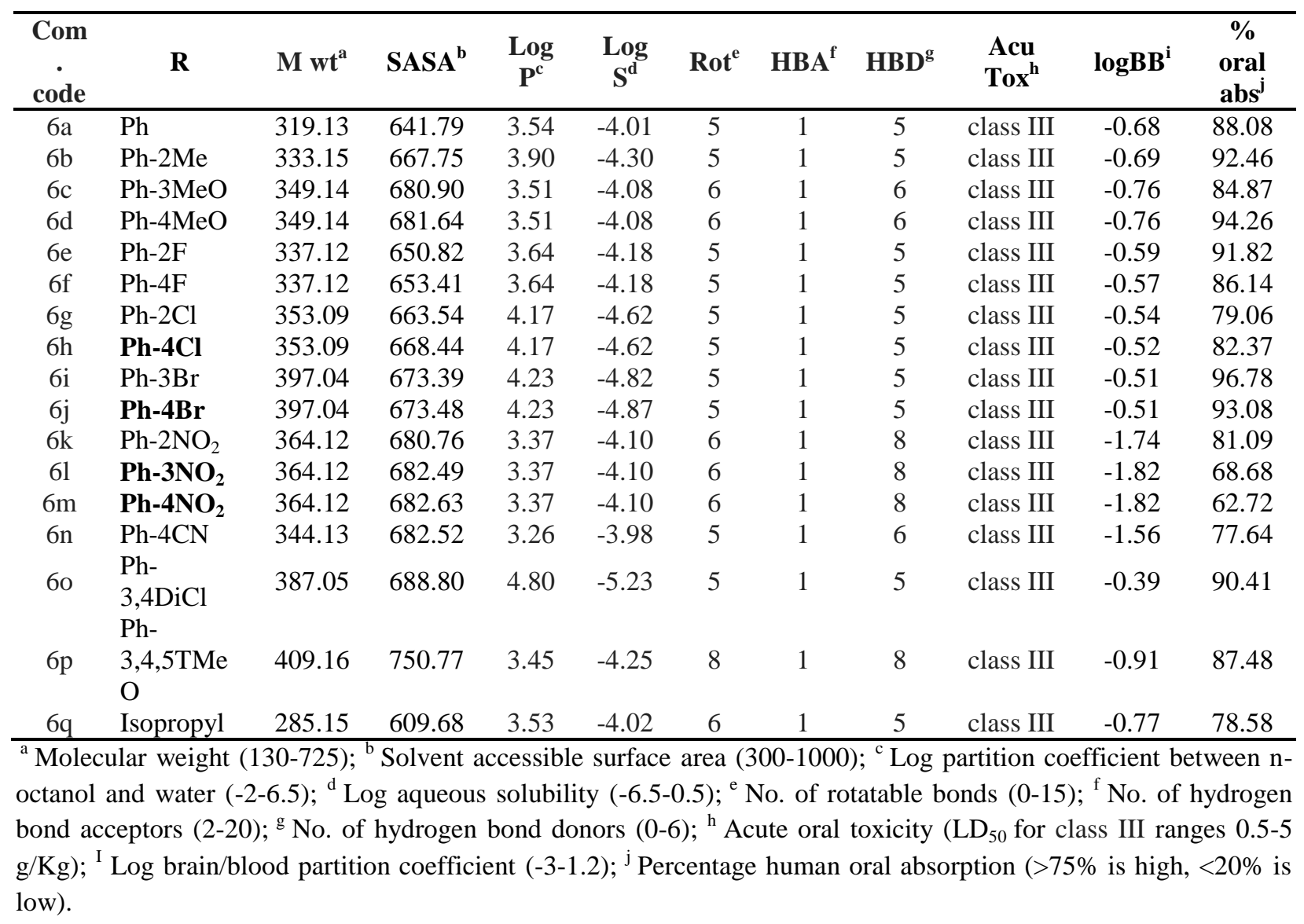

\section{Chemistry}


The synthetic route and optimized reaction conditions used for the designed compounds 6a-q, is illustrated in scheme 1. Synthesis of target compounds involved sequence of reactions, first step involved demethylation reaction of 6-methoxyquinoline (1) using Aluminum trichloride in dry DCM, which afforded intermediate 6-hydroxyquinoline (2). Reaction of intermediate 2 with ethyl 2-bromopropanoate was first tried under b1 conditions, but condition b1 not provided the complete conversion of $\mathbf{2}$ into $\mathbf{3}$ as per TLC, while under condition b2, complete conversion took place (as per TLC) and afforded the intermediate 3. In further step, intermediate $\mathbf{3}$ was treated with hydrazine hydrate which involved the replacement of ethoxy group with hydrazine and afforded the intermediate 4. In final step of scheme 1, to obtain the desired schiff base compounds (6a-q), intermediate $\mathbf{4}$ was condensed with specific aldehydes containing selected $\mathrm{R}$ groups (5a-q) to afford the titled compounds in good to excellent yield.

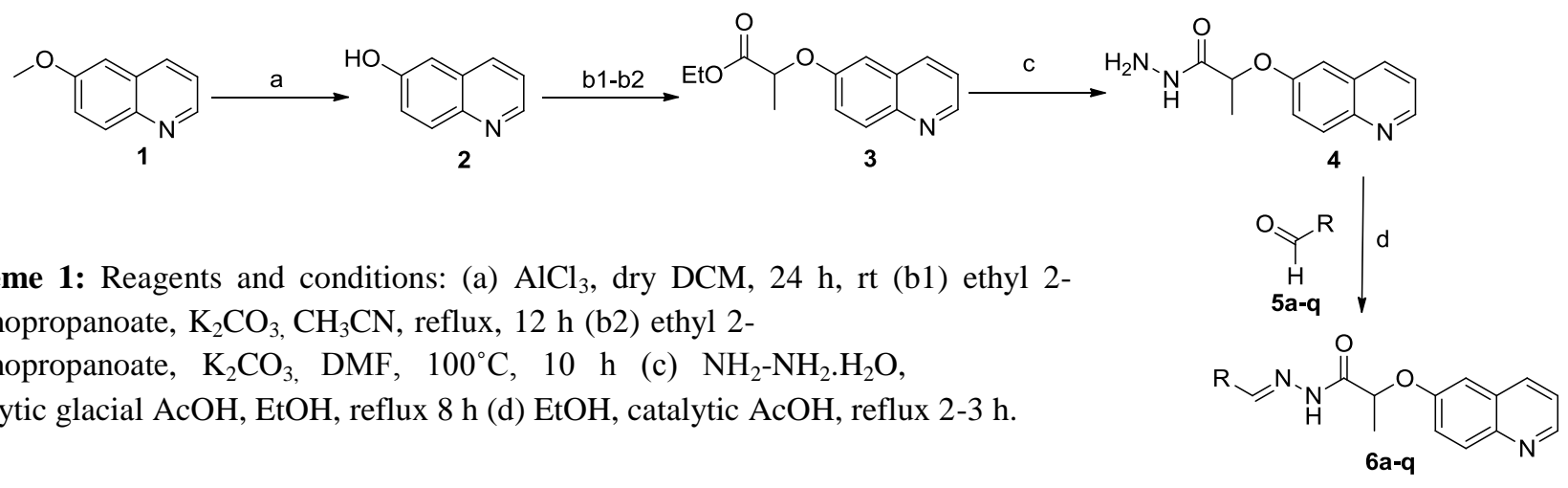

\section{Biological evaluation}

\section{Evaluation of anti-tubercular activity}

All purified compounds were evaluated for their inhibitory potency against H37Ra strain of $M$. Tuberculosis using luminometry method. The minimum inhibitory concentration (MIC) values (in terms of $\mu \mathrm{g} / \mathrm{mL}$ and $\mu \mathrm{M}$ ) of tested compounds and standard drug rifampicin are shown in Table 2. Among the tested compounds, four compounds $(\mathbf{6 h}, \mathbf{6 j}, \mathbf{6} \mathbf{\text { and }} \mathbf{6 m})$ displayed significant anti-Mtb activity with MIC $<20 \mu \mathrm{g} / \mathrm{mL}$ (highlighted by bold font in table 2). In further study, we investigated the effect of different substitution pattern of phenyl ring on anti-tubercular potency of compounds. Prototype compound 6a containing un-substituted phenyl ring exhibited weak anti-Mtb activity. Further, substitution of phenyl ring with electron donating groups like methyl and methoxy at ortho and meta position respectively, further decreased their anti-tubercular potency. Whereas, substitution by methoxy group at the para position (6d) slightly enhanced the 
potency $(38.51 \mu \mathrm{g} / \mathrm{mL})$. Further, substitution with strong electron withdrawing fluoro group at ortho and para position (compound 6e and $6 \mathbf{f}$ ) also results decreased in anti-tubercular potency (84.75 and $60.74 \mu \mathrm{g} / \mathrm{mL}$ respectively). Interestingly, chloro substitution at ortho position of phenyl ring $(\mathbf{6 g})$ markedly decreased the potency $(>180.78 \mu \mathrm{g} / \mathrm{mL})$, while at the para position (6h), potency against Mtb was significantly increased $(11.58 \mu \mathrm{g} / \mathrm{mL})$. Substitution with bromo at meta position (6i) not significantly changed the anti-tubercular potency, while para substituted compound $\mathbf{6 j}$ exhibited seven times more potency $(7.70 \mu \mathrm{g} / \mathrm{mL})$ as compared to the unsubstituted one. Among the nitro substituted compounds $(\mathbf{6 k}, \mathbf{6} \mathbf{l}$ and $\mathbf{6 m})$, compound $\mathbf{6 k}$ exhibited moderate while compounds $\mathbf{6 l}$ and $\mathbf{6 m}$ showed significant anti-tubercular potency. Over all anti-Mtb potency of compounds $\mathbf{6 k}$, $6 \mathbf{l}$ and $\mathbf{6 m}$ changed in the order ortho < meta < para (MIC 38.33, 14.27 and $7.13 \mu \mathrm{g} / \mathrm{mL}$ ) respectively. Further, 4-cyano substituted compound 6n exhibited moderate potency while di-chorlo and tri-methoxy substituted compounds ( 60 and $\mathbf{6 p}$ ) exhibited very weak anti-Mtb potency. Compound constitute isopropyl group instead of phenyl (6q), also exhibited weak anti-Mtb activity. So, overall SAR study of the tested compounds revealed that, anti-tubercular potency of compounds changed significantly with the position and nature of the substituent on phenyl ring. Compounds substituted with electron withdrawing group of moderate to large size at the para position of phenyl ring (especially $\mathbf{6 j}$ and $\mathbf{6 m}$ ) exhibited significant inhibitory potency against Mtb with good safety index.

In the recent studies, several carbohydrazide of heterocyclic moieties containing $N^{\prime}$-benzylidene at terminal part, are reported as potent inhibitor of pantothenate synthetase enzyme of $M$. tuberculosis (30, 31). Moreover, our reported compounds also exhibit the similar pharmacophoric features, so probably they may share the similar mechanism of action, but further studies are required to know the exact mechanism of action.

\section{Cytotoxicity evaluation}

All the synthesized compounds were evaluated for their cytotoxic effect on the MRC-5, human lung fibroblast cell at concentration of $512 \mu \mathrm{M}$. Interestingly, none of the compound showed toxicity at the tested concentration.

Table 2: Anti-Mtb and toxicity evaluation of the synthesized compounds

\begin{tabular}{llllll}
\hline $\begin{array}{c}\text { Comp } \\
\text { code }\end{array}$ & $\mathbf{R}$ & $\begin{array}{c}\text { MIC Mtb after } 7 \\
\text { days }(\boldsymbol{\mu M})\end{array}$ & $\begin{array}{c}\text { Toxicity } \\
\text { MRC5 } \\
(\boldsymbol{\mu M})\end{array}$ & $\begin{array}{c}\text { MIC in } \\
\boldsymbol{\mu g} / \mathbf{m L}\end{array}$ & $\begin{array}{c}\text { Selectivity } \\
\text { ratio }\end{array}$ \\
\hline $6 \mathrm{a}$ & $\mathrm{Ph}$ & 162.30 & $>512$ & 51.79 & $>3.15$ \\
\hline
\end{tabular}




\begin{tabular}{llllll}
\hline 6b & Ph-2Me & 234.60 & $>512$ & 78.15 & $>2.18$ \\
6c & $\mathrm{Ph}-3 \mathrm{MeO}$ & 258.40 & $>512$ & 90.21 & $>1.98$ \\
6d & $\mathrm{Ph}-4 \mathrm{MeO}$ & 110.30 & $>512$ & 38.51 & $>4.64$ \\
6e & $\mathrm{Ph}-2 \mathrm{~F}$ & 251.40 & $>512$ & 84.75 & $>2.03$ \\
6f & $\mathrm{Ph}-4 \mathrm{~F}$ & 180.20 & $>512$ & 60.74 & $>2.84$ \\
6g & $\mathrm{Ph}-2 \mathrm{Cl}$ & $>5120$ & $>512$ & $>180.78$ & $*$ \\
6h & $\mathbf{P h}-4 \mathrm{Cl}$ & $\mathbf{3 2 . 8 0}$ & $>\mathbf{5 1 2}$ & $\mathbf{1 1 . 5 8}$ & $>\mathbf{1 5 . 6 0}$ \\
6i & $\mathrm{Ph}-3 \mathrm{Br}$ & 141.30 & $>512$ & 56.10 & $>3.62$ \\
6j & $\mathbf{P h}-4 \mathrm{Br}$ & $\mathbf{1 9 . 4 0}$ & $>\mathbf{5 1 2}$ & $\mathbf{7 . 7 0}$ & $>\mathbf{2 6 . 3 9}$ \\
6k & $\mathrm{Ph}-2 \mathrm{NO}_{2}$ & 105.20 & $>512$ & 38.30 & $>4.86$ \\
61 & $\mathbf{P h}-\mathbf{3 N O}$ & $\mathbf{3 9 . 2 0}$ & $>\mathbf{5 1 2}$ & $\mathbf{1 4 . 2 7}$ & $>\mathbf{1 3 . 0 6}$ \\
6m & $\mathbf{P h}-4 \mathbf{N O}_{2}$ & $\mathbf{1 9 . 6 0}$ & $>\mathbf{5 1 2}$ & $\mathbf{7 . 1 3}$ & $>\mathbf{2 6 . 1 2}$ \\
6n & $\mathrm{Ph}-4 \mathrm{CN}$ & 87.90 & $>512$ & 30.24 & $>5.82$ \\
6o & Ph-3,4DiCl & 407.60 & $>512$ & 157.76 & $>1.25$ \\
6p & Ph-3,4,5TMeO & 422.70 & $>512$ & 172.95 & $>1.21$ \\
6q & Isopropyl & 291.20 & $>512$ & 83.03 & $>1.75$ \\
& Rifampicin & 0.18 & & 0.14 & \\
\hline
\end{tabular}

tested

\section{Conclusion}

In summary, we designed and synthesized seventeen (6a-q) novel quinoline based carboxylic hydrazides with anti-Mtb properties based on molecular hybridization as well as in-silico evaluated for drug likeness. Based upon the predicted in-silico physicochemical and pharmacokinetic parameters, all the compounds possessed drug likeness behaviour. Subsequently, compounds (6a-q) were tested to evaluate their cytotoxicity and whole cell activity against Mtb. Four compounds $(\mathbf{6 h}, \mathbf{6 j}, \mathbf{6}$, and $\mathbf{6 m})$ exhibited significant anti-Mtb activity (MIC $<20 \mu \mathrm{g} / \mathrm{mL}$ ), in which two most potent compounds (6j and $\mathbf{6 m}$ ) exhibited MIC values 7.70 and $7.13 \mu \mathrm{g} / \mathrm{mL}$ respectively against Mtb. None of the compound exhibited cytotoxicity against lung fibroblast cells at tested concentration $(512 \mu \mathrm{M})$. SAR study of the tested compounds revealed that, electron withdrawing group of moderate to large size especially at para position of phenyl ring (like $\mathbf{6 h}, \mathbf{6 j}$ and $\mathbf{6 m}$ ) significantly increased the potency against M. tuberculosis while retaining a good safety index. The tested compounds exhibited moderate potency against Mtb as compared to the reference drug rifampicin. We consider this study a helpful starting point 
for further lead optimization efforts aiming to find more potent anti-tubercular agents with an improved physicochemical profile.

\section{Acknowledgments}

Authors gratefully acknowledge BITS-Pilani for providing the necessary facilities to do this work. This work was carried out under a grant from Science and Engineering Research Board of Department of Science and Technology, New Delhi. (Ref. No: SR/FT/LS-58/2011). The work of DC and PC is funded by the FWO under grant agreement $12 \mathrm{~N} 5915 \mathrm{~N}$.

\section{Conflict of interest}

The authors declared that there is no conflict of interest

\section{References}

1. “"TUBERCULOSIS WHO Global Tuberculosis Report 2015” http://www.who.int/tb/Global_TB_Facts.pdf?ua=1 accessed on 26 Dec. 2015.

2. Esmail H., Barry C.E., Young D.B., Wilkinson R.J. (2014) The ongoing challenge of latent tuberculosis. Philos Trans R Soc Lond B Biol Sci;369:20130437.

3. Caminero, J.A., Sotgiu, G., Zumla, A., Migliori, G.B (2010) Best drug treatment for multidrug-resistant and extensively drug-resistant tuberculosis. Lancet Infect Dis;10:621629.

4. Calligaro G.L., Moodley L., Symons G., Dheda K. (2014) The medical and surgical treatment of drug-resistant tuberculosis. J Thorac Dis;6:186-95.

5. Multidrug and extensively drug-resistant TB (M/XDR-TB) 2010 Global Report on Surveillance and Response", WHO, Geneva, 2010.

6. Lange C., Abubakar I., Alffenaar J.W., Bothamley G., Caminero J.A., Carvalho A.C. (2014) Management of patients with multidrug-resistant/extensively drug-resistant tuberculosis in Europe: a TBNET consensus statement. Eur Respir J;44:23-63.

7. Parida S.K., Axelsson-Robertson R., Rao M.V., Singh N., Master I., Lutckii A. (2015) Totally drug-resistant tuberculosis and adjunct therapies. J Intern Med;277:388-405. 
8. Montales MT, Chaudhury A, Beebe A, Patil S, Patil N. HIV-Associated TB Syndemic: A Growing Clinical Challenge Worldwide. Front Public Health. 2015;3:281.

9. Kumar S., Bawa S., Gupta H. (2009) Biological activities of quinoline derivatives. Mini Rev Med Chem;9:1648-54.

10. Sharma R., Pandey A.K., Shivahare R., Srivastava K., Gupta S., Chauhan P.M.S. (2014) Triazino indole-quinoline hybrid: A novel approach to antileishmanial agents. Bioorg Med Chem Lett;24:298-301.

11. Chander S., Ashok P., Singh A., Murugesan S. (2015) De-novo design, synthesis and evaluation of novel 6,7-dimethoxy-1,2,3,4-tetrahydroisoquinoline derivatives as HIV-1 reverse transcriptase inhibitors. Chem Cent J;9:0111-6.

12. (2008) Handbook of anti-tuberculosis agents. Introduction. Tuberculosis;88:85-6.

13. Andries K., Verhasselt P., Guillemont J., Gohlmann H.W., Neefs J.M., Winkler H., Van Gestel J. (2005) A diarylquinoline drug active on the ATP synthase of Mycobacterium tuberculosis. Science;307:223-7.

14. Mahajan R. (2013) Bedaquiline: First FDA-approved tuberculosis drug in 40 years. Int J Appl Basic Med Res;3:1-2

15. Matteelli A., Carvalho A.C., Dooley K.E., Kritski A. (2010) Tmc207: The first compound of a new class of potent anti-tuberculosis drugs. Future Microbiol;5:849-58.

16. A. Zumla., P. Nahid., Cole S.T. (2013) Advances in the development of new tuberculosis drugs and treatment regimens. Nat Rev Drug Discov;12:388-404.

17. Rollas S., and Kücükgüzed S.G. (2007) Biological activities of hydrazone derivatives. Molecules;12:1910-39.

18. Rubbo S.D., Cymerman-Craig J. (1955) Anti-Tuberculous Activity of Verazide (1isoNicotinoyl-2-veratrylidene Hydrazine). Nature;176:887-887.

19. Lagorce D., Sperandio O., Baell J.B., Miteva M.A.,Villoutreix B.O. (2015) Faf-drugs3: A web server for compound property calculation and chemical library design. Nucleic Acids Res;43:(Web Server issue):W200-W207.

20. Qikprop. New York, Schrödinger, LLC, (2013) Version 3.8.

21. Cheng F., Li W., Zhou Y., Shen J., Wu Z., Liu G. (2012) Admetsar: A comprehensive source and free tool for assessment of chemical admet properties. J Chem Inf Model;52:3099-105. 
22. Negi A.S., Chattopadhyay S.K., Srivastava S., Bhattacharya A.K. (2005) A Simple regioselective demethylation of p-Aryl methyl ethers using aluminium chloridedichloromethane system. Synth Commun;35:15-21.

23. Roy K.K., Tota S., Tripathi T., Chander S., Nath C., Saxena A.K. (2012) Lead optimization studies towards the discovery of novel carbamates as potent ache inhibitors for the potential treatment of alzheimer's disease. Bioorg Med Chem;20:6313-20.

24. Rathore A., Rahman M.U., Siddiqui A.A., Ali A., Shaharyar M. (2014) Design and synthesis of benzimidazole analogs endowed with oxadiazole as selective COX-2 inhibitor. Arch Pharm;347:923-35.

25. Zhang F., Wang X.L., Shi J., Wang S.F., Yin Y., Yang Y.S. (2014) Synthesis, molecular modeling and biological evaluation of n-benzylidene-2-((5-(pyridin-4-yl)-1,3,4oxadiazol-2-yl)thio)acetohydrazide derivatives as potential anticancer agents. Bioorg Med Chem;22:468-77.

26. Snewin V.A., Gares M.P., Gaora P.O., Hasan Z., Brown I.N., Young D.B. (1999) Assessment of immunity to mycobacterial infection with luciferase reporter constructs. Infect Immun;67:4586-93.

27. Jones PA, King AV. (2003) High throughput screening (HTS) for phototoxicity hazard using the in vitro 3T3 neutral red uptake assay. Toxicol In Vitro. 17:703-08.

28. Qikprop, User Manual (2014), Schrödinger, LLC, Chapter-1, Version 4, 2-5.

29. Jorgensen W.L., Duffy E.M. (2000) Prediction of drug solubility from Monte Carlo simulations. Bioorg Med Chem Lett;10:1155-8.

30. Samala G., Nallangi R., Devi P.B., Saxena S., Yadav R., Sridevi J.P., Yogeeswari P., Sriram D. (2014) Identification and development of 2-methylimidazo [1,2-a]pyridine-3carboxamides as Mycobacterium tuberculosis pantothenate synthetase inhibitors. Bioorg Med Chem;22:4223-32.

31. Samala G., Devi P.B., Saxena S., Meda N., Yogeeswari P., Sriram D. (2016) Design, synthesis and biological evaluation of imidazo[2,1-b]thiazole and benzo[d]imidazo[2,1b] thiazole derivatives as Mycobacterium tuberculosis pantothenate synthetase inhibitors. Bioorg Med Chem;24:1298-307. 